\title{
Quality Control of the Atmospheric Forcing Data in the Operational System of the Black Sea Marine Forecasting Center
}

\author{
Yu.B. Ratner, M.V. Ivanchik, A.M. Ivanchik, A.L. Kholod \\ Marine Hydrophysical Institute, Russian Academy of Sciences, Sevastopol, Russian Federation \\ e-mail:yubrat@gmail.com
}

\begin{abstract}
Problems of reliability control of the atmospheric forcing data used for setting boundary conditions in the model simulations performed in the Black Sea marine forecasting center (BS MFC) of MHI (RAS) are considered. To set the boundary conditions on the sea surface it is necessary to have information about the heat, momentum, evaporation and precipitation total fluxes and short-wave radiation penetrating in the upper sea layer with a thickness of about fifty meters. To check the reliability of the data obtained the information not only about these fluxes, but also about their components appears to be of high value. The first section describes the weather forecasts applied in BS MFC for the atmospheric boundary layer. The methods for controlling the weather forecast reliability of the atmosphere boundary layer are considered. The software developed to solve this task is briefly described in the second section. The first group of control methods is based on the calculation according to the approximate formulas short-wave and long-wave radiation fluxes. For other components of total fluxes their comparison according to the forecast data of two atmospheric models used in BSMFC to set the boundary conditions on the sea surface is used. The third section contains the examples of the errors occurring in preparing the weather forecast data. Errors in the data on the magnitude of the albedo and upward short-wave radiation fluxes were discovered on the basis of the application of the developed methods. The conclusions drawn from the results obtained testify to effectiveness of the system developed to control data reliability. Further investigations are planned. It is noted that the results represented in the paper constitutes a basis for development of the software intended to correct the revealed errors.
\end{abstract}

Keywords: marine forecasts, atmospheric forcing, boundary conditions, heat flows, evaporation, precipitation, long-wave radiation, short-wave radiation, albedo, Stefan - Boltzmann constant, reliability.

DOI: $10.22449 / 1573-160 X-2016-5-24-35$

(C) 2016, Yu.B. Ratner, M.V. Ivanchik, A.M. Ivanchik, A.L. Kholod

(C) 2016, Physical Oceanography

Introduction. Within 2009 - 2014 Marine Hydrophysical Institute (MHI) participated in EC FP7 Project MyOcean as one of the seventh marine forecasting centers. Currently, the center continues to operate independently. One of the tasks of further improvement of the operating system of the Black Sea marine forecasting center (BSMFC) is associated with the creation of quality control and reliability of the atmosphere boundary layer condition (atmospheric forcing) data. This data is used to specify the boundary conditions at the sea surface performing diagnostic and prognostic calculations by means of the Black Sea circulation model.

The purpose of this paper is to describe the principles and methods assumed as a basis of the quality control module being developed, as well as brief information on the developed version of the software and an illustration of reliability of the atmospheric forcing data testing results obtained by MHI BS MFC. This data comes from two sources:

- SKIRON Meteorological Center (Greece) [1];

- System of regional weather forecasts of MHI Ocean-Atmosphere Interaction (OAI) department [2]. 
1. Atmospheric forcing data used for setting boundary conditions in the marine diagnostic and prognostic calculations

Data on the atmospheric boundary layer condition must be known to the grid nodes of a physical model of the marine environment on the sea surface, possess a high spatial resolution and accuracy appropriate to the solving problems of marine forecasts. Such data can be obtained only as a result of prognostic calculations based on the modern atmospheric circulation models. SKIRON Meteorological Center applies Eta model [3], the OAI Department applies MM5 [4].

Owing to the complexity of modern atmospheric models, various kinds of interferences and disruptions may appear in the obtained atmospheric forcing fields. The data processing centers, preparing the products we are interested in, take into account this phenomenon and provide the quality control measures. However, there are occasionally situations when the existing system, by virtue of its imperfections, is not operating properly.

To solve this problem within the framework of MyOcean project the recommendation for the further control of incoming input data was issued. It was advisable to stick to during to the current operation of the center of the MHI Black Sea marine forecasting center.

Reliability control methods considerably depend on the specific characteristics of the input data. Before our proposed methods to control the reliability of atmospheric forcing data are recounted, it is necessary to underline that to perform the marine forecasts for the applied Black Sea circulation model [5] input, the following information should be obtained:

- total heat flux from the atmosphere to the sea;

- the short-wave radiation flux penetrating into the upper layers of the sea to a depth of several tens of meters;

- the evaporation flux from the sea surface to the atmosphere (evaporation);

-precipitation flux in the form of rain or snow falling on the sea surface of a unit area per time unit;

- momentum flux between the atmosphere and the sea (zonal and meridional wind stress components.

The applied data of the forecasts by atmospheric models do not directly contain some of these values. These are the sets of other atmospheric parameters with a maximum prognostic period of 5 days and the return periodicity of results of 1 hour.

Digital sets of the SKIRON data contain the results of forecasts of twodimensional fields of atmospheric parameters. These data are the GRIB format files [6] and include the following parameters

- zonal and meridional wind velocity components at $10 \mathrm{~m}$ above sea level;

- air temperature and specific humidity at $2 \mathrm{~m}$ above sea level;

- cloud cover, cloudiness, cloud amount;

- air pressure above sea level;

- precipitation;

- downward short-wave radiation flux (downward short-wave radiation) in the atmosphere in the range of $0.4-4$ microns reaching the sea surface;

- upward short-wave radiation flux (upward short-wave radiation) in the atmosphere at sea level, which is precisely up to $1-2 \%$ equal to downward shortwave radiation scattered by the sea surface;

PHYSICAL OCEANOGRAPHY NO. 5 (2016) 
- downward long-wave radiation flux (downward long-wave radiation) in the atmosphere in the range of $4-13$ microns reaching the sea surface;

-upward long-wave radiation flux of the sea surface in the range of $4-13$ microns;

- evaporation (flux);

- latent heat flux (gained or lost by the sea due to the phase transitions of water by evaporation and precipitation);

- sensible heat flux (gained or lost by the sea due to the contact turbulent heat exchange between the air and sea water at the sea surface);

- sear surface temperature.

This set of parameters permits both to calculate the required boundary conditions for the forecast on the hydrodynamic model of the sea and also quite in detail to check the correctness of the data obtained by atmospheric forcing.

Digital sets of the OAI Department data are the NetCDF format files and contain the following parameters:

- air temperature and specific humidity at $2 \mathrm{~m}$ above sea level;

- sear surface temperature;

- zonal and meridional wind velocity components at $10 \mathrm{~m}$ above sea level;

- sensible heat flux;

- latent heat flux;

- downward short-wave radiation flux reaching the sea surface;

- upward short-wave radiation flux at sea level;

- downward long-wave radiation in the atmosphere reaching the sea surface;

- upward long-wave radiation flux of the sea surface;

- zonal and meridional wind velocity components;

- precipitation intensity (height of the water layer formed per time unit, provided that the water does not spread on the surface and is accumulated, such as in the rain gauge).

Unlike the first atmospheric forcing data source, the aforementioned set of parameters do not permit to control their quality with that degree of completeness, which is possible for the data [3], as there are no atmospheric pressure and cloudiness data. However, if under the data quality control [4] the results obtained during the data check [3] for the same dates are used, the marked obstacle is substantially leveled.

\section{Test system and software used to control the atmospheric forcing} reliability

The above information permits to proceed to the consideration of the principles and methods of the control of atmospheric forcing data reliability. First of all, it the need to control comprehensive set of three-dimensional fields (two spatial dimensions and one temporal) should be noted. Even applying the modern information technologies it is a rather time-consuming process. That is why many existing control systems use a simplified approach based on a step-by-step solution of the problem. At that the data control starts with the time series spatial variability analysis of mean values of atmospheric parameters over the selected sea surface area. The more complex procedures of analysis of two- and three-dimensional fields are carried out in the following stages, taking into account the results of the primary control. This approach is implemented in the quality control systems of 
thematic centers (TAC - Thematic Assembling Center) of Copernicus (European system of the marine forecasts) [7] input data. The result of the trial operation of such systems showed that most of the problems were revealed during the first simplified control stage. In view of the foregoing, it was the development of methods and software for the first stage of data quality control the development of the own system began precisely with.

Data quality control system should consist of a set of interrelated tests having different complexity levels created to control certain groups or separate parameters of atmospheric forcing. All the software that implements tests to control the data reliability is worthwhile to be developed in the statistical computing environment $R$ [8], which has an integrated suite of software facilities for data manipulation, calculation and graphical display. The advantage of $R$ environment is the possibility of free (without having to buy a license) use.

Below we are to consider a group of tests, which can be offered to control the reliability of prognostic values of the radiation fluxes derived according to atmospheric models.

One of the simplest tests (TULWR), which we have implemented, is aimed at controlling of the upward long-wave radiation data flux reliability. Based on the known physical laws of thermal radiation (Kirchhoff's and Stefan - Boltzmann laws) and assuming that the sea surface in this wavelength range of the electromagnetic spectrum radiates as an black body, we can write the following ratio for the upward long-wave radiation flux

$$
\frac{Q_{U L W R}}{T^{4}}=\sigma,
$$

where $Q_{U L W R}$ is an upward long-wave radiation flux spreading up from the sea surface, $\mathrm{W} / \mathrm{m}^{2} ; T$ is the sea surface temperature, $\mathrm{K} ; \sigma=5.670 \cdot 10^{-8}$ is the Stefan Boltzmann constant, $\mathrm{W} / \mathrm{m}^{2} / \mathrm{K}^{4}$.

Having calculated this ratio for each node of the atmospheric model twodimensional grid and averaged the values obtained for the nodes of marine waters, we can get time series values $\hat{\sigma}$. Comparing the relative values of the differences $(\hat{\sigma}-\sigma) / \sigma$ with the known threshold value of $1-2 \%$ and assuming that there are no failures in the sea surface temperature values, a conclusion about the quality of the upward long-wave radiation data flux $Q_{U L W R}$ can be drawn.

Tests for the data control of downward long-wave radiation data are feasible in the absence of clouds. They require quite a precise knowledge of the vertical profiles of pressure, temperature, humidity and other parameters of the atmosphere. For their realization it is necessary to use complex radiation models. In view of this, we are not involved in development of such kind of tests. However, taking into account the presence of two sources of atmospheric forcing data at our disposal, we can compare them. For large differences in the values of radiation fluxes, e.g. more than $30 \%$, we can assume the presence of invalid data in one of the available sources.

The test (TDSWR) considered in the present paper is applied to control the reliability of the values of short-wave solar radiation fluxes in clear atmosphere. It is more complicated and is based on the use of propagation models of optical 
radiation in the atmosphere. Such models are described in [9-12]. The functions included in the INSOL library [13] of the $\mathrm{R}$ statistical package, allow the performance of these calculations. Also, the following information is required: data on the specific humidity and air temperature at the sea surface, the atmospheric ozone concentration and visibility range, the sea surface albedo, the solar zenith angle and the Julian date.

Data on the ozone concentration in the atmosphere, visibility range and the sea surface albedo are absent in sets of output parameters of the both atmospheric models. Information on the ozone concentration and visibility range can be obtained from the climate data archives. Information on the sea surface albedo can be set in accordance with $[14,15]$ etc. The aforementioned data are rather diverse, due to the strong variability of the albedo depending on the specific meteorological conditions, primarily due to changes in the solar zenith angle the sea surface roughness, associated with the wind.

Having both time series of downward short-wave radiation values $\hat{Q}_{D S W R}$ calculated on the basis of the optical atmospheric model [13] and averaged over areas of the sea surface, which are not a covered by cloudiness, and time series $Q_{D S W R}$ obtained in a similar manner from the output of the model ([3] or [4]), it is possible to plot the graphs and compare the statistical characteristics of the deviation between the time series, thereby getting an idea about the data quality $Q_{D S W R}$.

TUSWR test is assigned for quality control of the data of upward short-wave radiation flux in cloudless conditions. It can be implemented if the sea surface albedo is set according to the aforementioned references. In this case it is possible to calculate the averaged over space upward short-wave radiation fluxes $\hat{Q}_{U S W R}$ and compare them with the similarly averaged values $Q_{U S W R}$ obtained from the output of the atmospheric models based on the use of data $Q_{D S W R}$, put through TDSWR test, and information on the albedo.

Due to the lack of all necessary information and inaccuracy of the used optical atmospheric model, there may be significant differences between the values of the compared values obtained from the output of the atmospheric models and the ones calculated using the INSOL library [13], while performing both tests (TDSWR and TUSWR). Therefore, it is necessary to give a more precise clarification of the thresholds for the lower and upper boundaries of the difference values of the compared values. This should be done in such a way that at the overrun of these boundaries the fluxes resulting from the forecast by the atmospheric models were likely to contain pieces of invalid data. Currently, we do not apply these tests and their development is far from being completed.

The problem of determining the values of the albedo can be resolved through the use of the following relation

$$
\alpha=Q_{U S W R} / Q_{D S W R},
$$

where $\alpha$ is the sea surface albedo; $Q_{U S W R}$ is the upward short-wave radiation flux; $Q_{D S W R}$ is the downward short-wave radiation flux. 
Thus, it is possible directly to compare the value $\alpha$ calculated by formula (2) with the data of the references. Then the considered test, denoted as $A L B$, becomes complex test of simultaneous reliability control of the upward and downward atmospheric radiation data and the sea surface albedo. However, in this case the interpretation of the results will be more complicated. Also, it is necessary to define what data $-Q_{U S W R}$ or $Q_{D S W R}-$ is actually unreliable.

The aforementioned tests are intended to control the reliability of radiation heat flows. Control of turbulent fluxes may be implemented applying bulkformulas. This method permits to control data on the momentum flux, sensible and latent heat. One of the latest variants of such, given in [16], is used. These are the very design ratios which are implemented in $N E M O$, one of the modern ocean models [17].

On the basis of the given approach the software for calculation, analysis and display of the test criteria statistical characteristics used for atmospheric forcing quality control was developed. Its development is performed using the statistical computing environment $R$ [8]. This software control tools provide:

- calculation of the TULWR and ALB criteria values, averaged over the Black Sea water area and used to control the reliability data on the short-wave and longwave radiation fluxes;

- calculation of the heat flow values according to the bulk-formulas, given in [16];

- averaging of two-dimensional spatial fields of the parameters of atmospheric boundary layer state, the momentum fluxes, heat and humidity on the current forecast period;

- visualization of time-series value graphs used for quality control of heat flows and atmospheric boundary layer parameters.

3. Results of atmospheric forcing data quality control of SKIRON and MHI Ocean-Atmosphere Interaction Department

3.1. Upward and downward long-wave radiation control. The upward longwave radiation flux is one of the components of the heat flow. It can be controlled based on the calculation according to the formula (1). The SKIRON data control results are shown in Fig. 1.

In TULWR test time series graphs for 2009 - 2010 years (Fig. 1, $a, b$ ) blue color shows the mean value of $\hat{\sigma}$ obtained for each day of the year according to the forecast data for 9:00 Coordinated Universal Time (UTC). Two red curves situated above and below the $\hat{\sigma}$ time series graph correspond to the minimum and maximum values of the relation in the right part of the formula (1). These values were calculated over the entire Black Sea water area. In 2011 a single failure is detected in the $\hat{\sigma}$ time series graph shown in Fig. 1. Value $\hat{\sigma}$ differs significantly from $\sigma$. For the 2012 year time series a whole interval of time at the end of the year, where the value $\hat{\sigma}$ is significantly different from $\sigma$, is found (Fig. 1, $d$ ). Fig. $1, d$, e shows the $\hat{\sigma}$ time series for 2013 and 2014. It can be seen that the time intervals of significant differences between values $\hat{\sigma}$ and $\sigma$ increase even more as compared with 2012. 

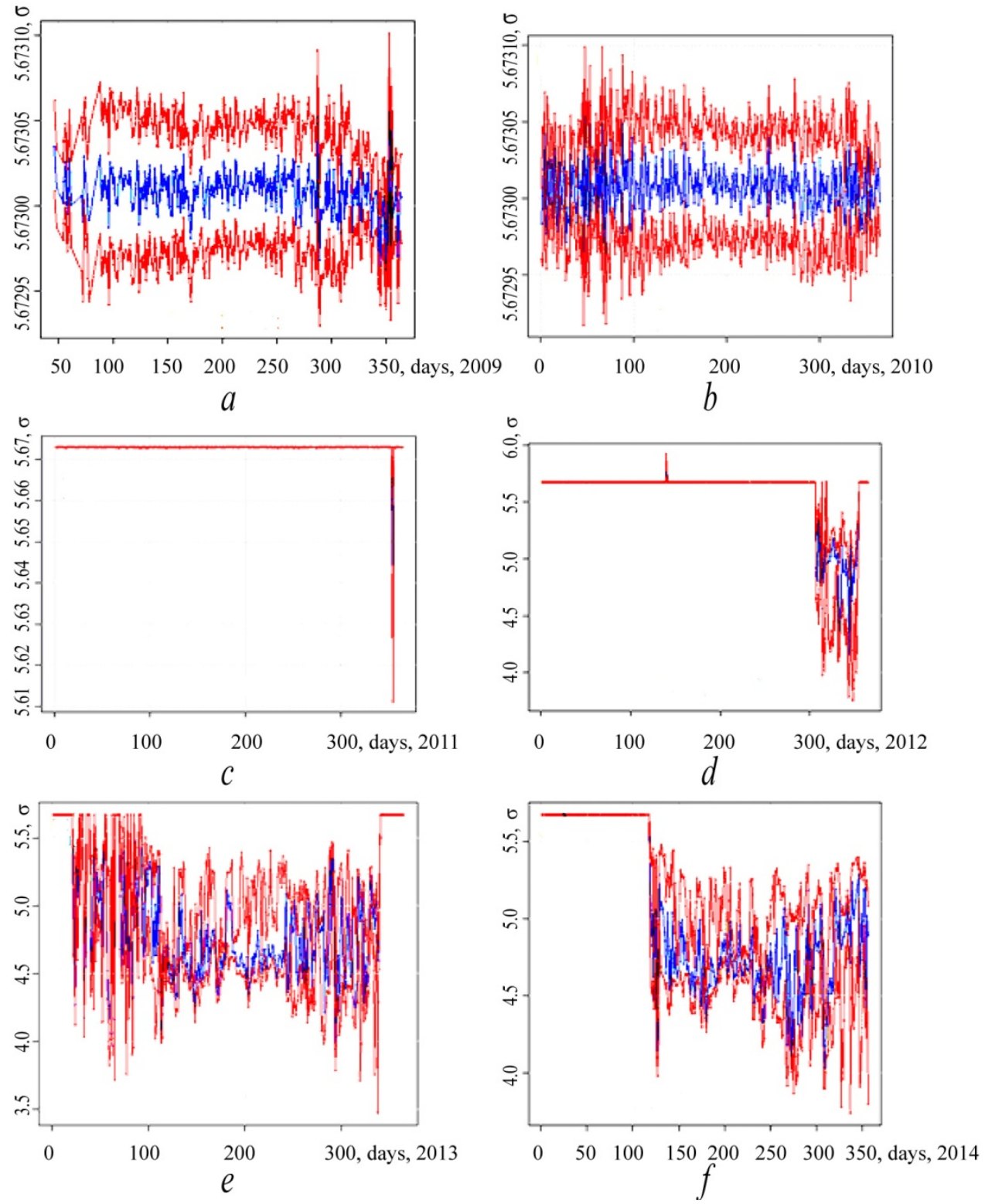

Fig. 1. $Q_{U L W R}$ calculation correctness control according to the criterion $\sigma=Q_{U L W R} / S S T^{4}: a-$ according to the data of 2009, $b$ - according to the data of 2010, $c$ - according to the data of 2011, $d-$ according to the data of 2012, $e$ - according to the data of 2013, $f$ - according to the data of 2014.

The discovered peculiarities allow for the conclusion that the prognostic values of upward and downward long-wave radiation fluxes for 2012, 2013 and 2014 may contain large amounts of incorrect data. Further examination of these peculiarities was continued on the basis of analysis of time series graphs of upward and downward long-wave radiation fluxes $-Q_{D L W R}$ and $Q_{U L W R}$. Analysis of the time series showed that when $\hat{\sigma}$ is substantially different from $\sigma$, the values $Q_{U L W R}$ are calculated correctly. This is confirmed by the graphs of $Q_{D L W R}$ and 
$Q_{U L W R}$ values for 2014 (Fig. 2). A similar situation took place both in 2012 and 2013.
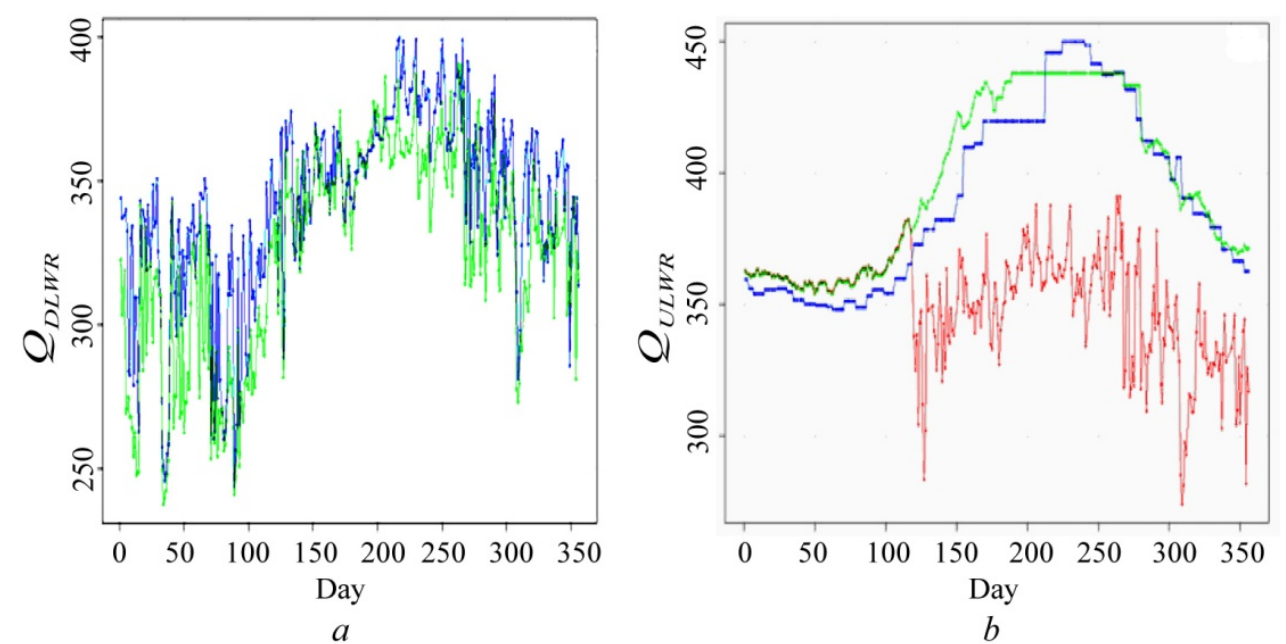

Fig. 2. $Q_{D L W R}$ and $Q_{U L W R}$ time series in accordance to the SKIRON and OAI forcing and the StefanBoltzmann law: $a-Q_{D L W R}, b-Q_{U L W R}$

In Fig. 2, a green color marks the flux values obtained by the SKIRON data, the blue one - by OAI Department data. The values $Q_{D L W R}$ obtained by the SKIRON and OAI data comply with both each other with an accuracy characteristic of modern calculation methods of these quantities for different weather models and existing theoretical ideas on the nature of the atmospheric downward long-wave radiation intra-annual variability.

In Fig. 2, $b$ red color marks the flux values $Q_{U L W R}$ obtained by the SKIRON data, the blue one - by OAI Department data, the green - the values calculated according to the Stefan-Boltzmann law by formula (1).

In contrast to the aforementioned case, there are significant differences between the values of the fluxes $Q_{U L W R}$ obtained according to various sources. Noteworthy is the sharp difference between the variability nature of time series values obtained by the SKIRON data from those obtained from other sources. Till the end of April the nature of changes in all three $Q_{U L W R}$ time series is about the same. Further the values $Q_{U L W R}$ corresponding to the SKIRON data are sharply different from those obtained by other data sources. Moreover, $Q_{U L W R}$ variability nature, according to SKIRON, does not correspond to the existing theoretical assumptions about the intra-annual variability of upward long-wave radiation fluxes of the sea surface - atmosphere system. Comparing the graphs in Fig. 1, $f$ PHYSICAL OCEANOGRAPHY NO. 5 (2016) 
and 2, $b$, it can be seen that the changes in the behavior of the time series $Q_{U L W R}$ and $\hat{\sigma}$ are synchronous. The marked peculiarities give occasion to the conclusion that the upward long-wave radiation fluxes of the sea surface-the atmosphere system have been incorrectly calculated in SKIRON Meteorological Center.

The graphs of $Q_{U L W R}$ values shown in Fig. 2, $b$, also allow to draw a conclusion about the possible low quality of the sea surface temperature data used for weather forecasts in SKIRON Meteorological Center and OAI Department. This is evidenced by the stepped variability nature of $Q_{U L W R}$ values, especially as for the OAI data. According to numerous research of the intra-annual Black Sea surface temperature variation, such stepped peculiarities should not take place. In addition, there are time delay $Q_{U L W R}$ changes found in the OAI data respectfully to that which should actually be observed.

On the basis of the test results OAI Department started to use the SST prognostic fields obtained by the BS MFC data as the values for weather forecasts. As a result, the problems noted in 2015 are no longer observed.

3.2. Control of upward and downward short-wave radiation fluxes. Control of upward and downward short-wave (direct and scattered) radiation fluxes was carried out on the basis of direct comparison of $Q_{D S W R}$ values calculated according to the SKIRON and OAI data. Time series of values of these fluxes for 2011 - 2014 are showed in Fig. 3, $a-d$. In general, the variability nature of the compared time series is the same for both data sources and corresponds to the available theoretical concepts on annual variability of solar radiation fluxes. The observed differences can be explained by distinctions of the calculation algorithms of cloudiness and aerosol characteristics by models [17] and [18]. Aerosol affects the short-wave solar radiation scattering and, as a result, the fluxes of this radiation incident on the marine surface.

Under conditions when there is no cloud and downward short-wave radiation flux data can be considered reliable, the control of the reliability of data on the magnitude of the albedo and upward short-wave radiation fluxes can be made on the basis of the $A L B$ test. Graphs of time series values of the albedo for cloudless conditions, averaged over the Black Sea water area and calculated according to the SKIRON data for 2011 - 2014 are shown in Fig. 4. 

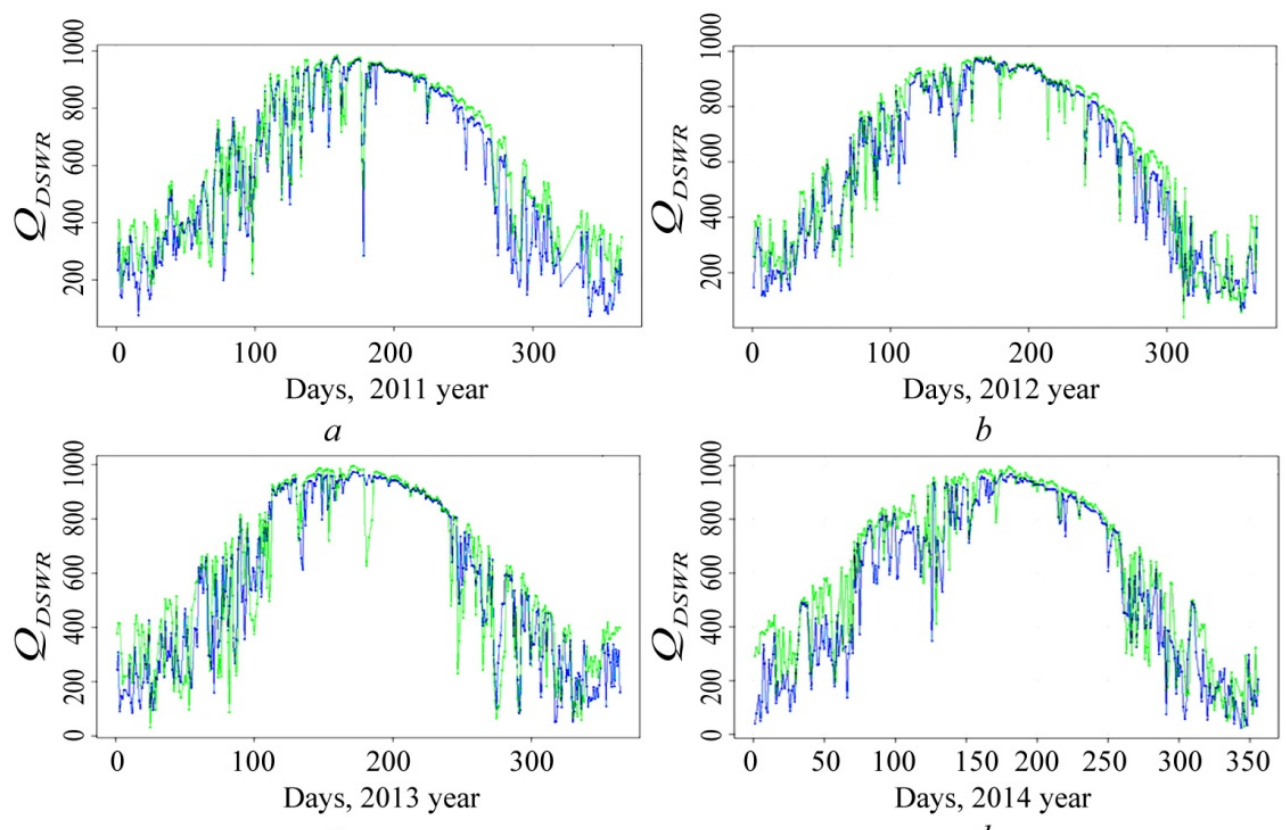

$c$

$$
d
$$

Fig. 3. Q Q data for 2011, b-QDSWR according to the data for 2012, c- QDSWR according to the data for 2013, dQDSWR according to the data for 2014.
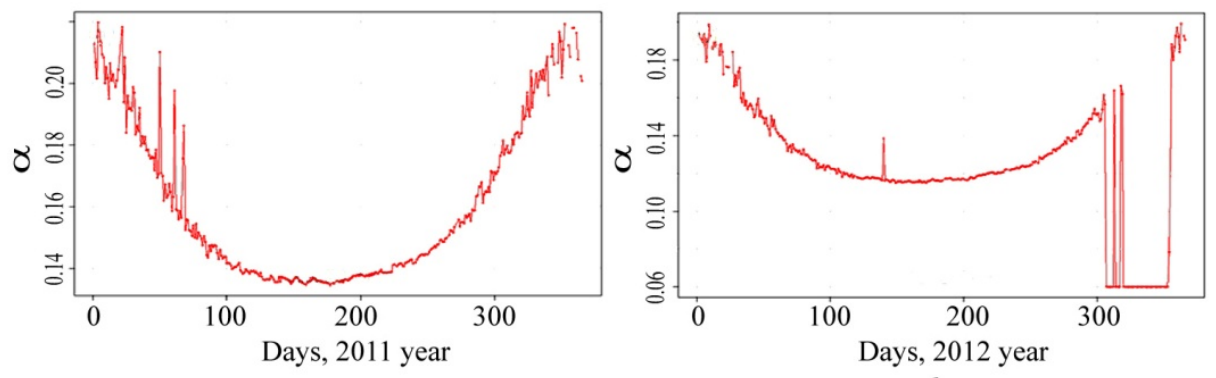

$a$

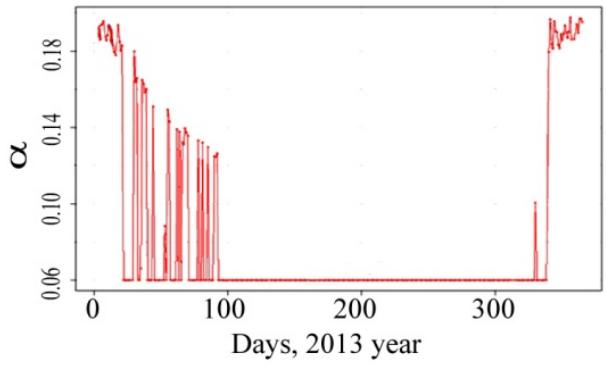

$c$

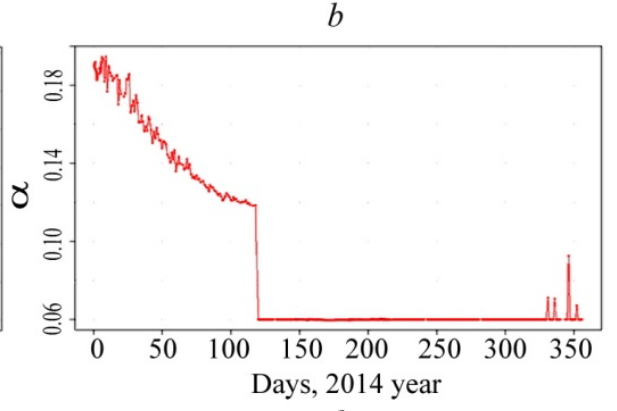

d

Fig. 4. Control of calculation correctness of $Q_{U S W R}$ and $Q_{D S W R}$, obtained from the SKIRON data sources, according to the albedo value $\alpha=Q_{U S W R} / Q_{D S W R}: a$ - albedo according to the data for 2011, $b$ albedo according to the data for 2012, $c$ - albedo according to the data for 2013, $d$ - albedo according to the data for 2014

PHYSICAL OCEANOGRAPHY NO. 5 (2016) 
Albedo depends on the height (zenith angle) of the Sun [14] and reaches its maximum at noon local time at $34^{\circ} \mathrm{E}$ every day of the year. Due to the relatively small size of the Black Sea the albedo values do not change greatly over the space. At the same time, due to seasonal changes in the height of the Sun, the value $\alpha$ at the noon local time, approximately corresponding to 9:00 UTC, reaches its maximum during the winter solstice and the minimum - during the summer one. With the change of season, the value of albedo gradually decreases from the maximum to a minimum during the transition from winter to summer and increases during the transition from summer to winter. This is the nature of the albedo temporal variability, calculated according to the formula (2). It is demonstrated on the graph shown in Fig. 4, a (excluding emissions associated with the inaccuracy of the data due to the cloudiness). At the same time for 2012 - 2014 the time dependence of the albedo values is a completely different, inadequate to the physical reality. It is marked by the extended periods of time, when after a sharp fall of albedo to the value $\alpha \approx 0,06$ it remains constant, approximately corresponding to the values used in $[4,16]$. These changes are synchronized with the corresponding peculiarities of the observed time series $\hat{\sigma}$ and $Q_{U L W R}$ calculated on the basis of the SKIRON data use for 2012, 2013 and 2014. Consequently, upward short-wave radiation fluxes near the sea surface, obtained according to the SKIRON data, could also be unreliable. In addition, the obtained minimum albedo values in 2011 are approximately 1.5 - 2 times higher than the values mentioned in the references [14].

Conclusions. Application of the suggested system of tests and software developed on their basis, permitted to reveal inaccuracies and errors in the atmospheric forcing data provided by SKIRON Meteorological Center and OAI Department. These marked failures led to a decrease in the reliability of marine diagnostic and prognostic calculations in the Black Sea water area within the period from the end of 2012 to 2014. Further development of the atmospheric forcing reliability control system is planned to involve the development of more sophisticated tests for the control of short-wave radiation and downward long-wave atmospheric radiation fluxes. Special software for correction of the upward radiation fluxes has been developed on the basis of the results obtained. It permits to improve the accuracy of marine forecasts applying the available atmospheric forcing data.

\section{REFERENCES}

1. http://forecast.uoa.gr

2. http://www.hydrophys.ru

3. Papadopoulos, A., Katsafados, P. \& Kallos, G. [et al.], 2002, "The weather forecasting system for Poseidon - an overview”, The Global Atmosphere and Ocean System, vol. 8, no. 2-3, pp. 219-237.

4. Barabanov, V.S., Efimov, V.V. \& Shokurov, M.V., 2003, "Ob ispol'zovanii mezomasshtabnoy modeli dlya rascheta privodnogo vetra i osadkov nad Chernym morem [On the use of a mesoscale model for the calculation of the near-water wind and precipitation over the Black Sea]”, Ekologicheskaya bezopasnost' pribrezhnoy i shel'fovoy zon i kompleksnoe ispol'zovanie resursov shel'fa, iss. 7, pp. 177-189 (in Russian).

5. Demyshev, S.G., 2012, "Chislennaya model' operativnogo prognoza techeniy $v$ Chernom more [Numerical model of the operational forecast of currents in the Black Sea]", Izv. RAN. Fizika atmosfery i okeana, vol. 48, no. 1, pp. 137-149 (in Russian). 
6. 2003, "Introduction to GRIB Edition 1 and GRIB Edition 2", World Meteorological Organization, 8 p.

7. http://www.copernicus.eu/

8. http://www.R-project.org/

9. Iqbal, M., 1983, “An Introduction to Solar Radiation”, Orlando, Academic Press, 407 p.

10. Hulstrom, R., Bird, R. \& Riordan, C., 1985, "Spectral solar irradiance data sets for selected terrestrial conditions”, Solar Cells, vol. 15, no. 4, pp. 365-391.

11. Freidenreich, S.M., Ramaswam, V., 1999, "A new multiple-band solar radiative parameterization for general circulation models”, J. Geophys. Res., vol. 104, no. D24, pp. 31389-31409.

12. Warner, John C., Armstrong, Brandy \& He, Ruoying [et al.], 2010, "Development of a Coupled Ocean-Atmosphere-Wave-Sediment Transport (COAWST) Modeling System”, Ocean Model., no. 35, pp. 230-244.

13. https://cran.r-project.org/web/packages/insol/index.html

14. Kondrat'ev, K.Ya., Binenko, V.I., D'yachenko, L.N. [et al.], 1981, “Al'bedo i uglovye kharakteristiki otrazheniya podstilayushchey poverkhnosti i oblakov [Albedo and angular characteristics of reflection of the underlying surface and clouds]", Leningrad, Gidrometeoizdat, 232 p. (in Russian).

15. Sayer, A., 2007, “A sea surface reflectance model Suitable for use with AATSR aerosol retrieval”, AOPP Memorandum University of Oxford, 31 p.

16. Large, W.G., Yeager, S.G., 2004, "Diurnal to Decadal Global Forcing For Ocean and Sea-Ice Models: The Data Sets and Flux Climatologies”, NCAR/TN-460+STR NCAR TECHNICAL NOTE, 105 p.

17. 2014, "Gurvan Madec and the NEMO team. NEMO ocean engine. Version 3.6", France, Institut Pierre-Simon Laplace, 382 p.

18. Lazic, L., Telenta, B., 1990, "Documentation of the UB/NMC (University of Belgrade and National Meteorological Centre, Washington) Eta Model. Tropical Meteorology Research Programme”, World Meteorological Organization, Geneve, WMO/TD, no. 366, 304 p. 